\title{
Acoustic and thermal vibrational behavior of rare earth glasses
}

\begin{abstract}
The ultrasonic wave velocity and the thermal expansion of the rare earth glasses have been measured as functions of temperature and pressure to test predictions of the soft potential model for the acoustic and thermal properties. The longitudinal ultrasonic wave velocities increase under pressure. The hydrostatic pressure derivative of the bulk modulus is positive: these glasses show a normal elastic response as compressed. However, the pressure derivative of the shear modulus is negative and small, indicating weak softening of shear modes under pressure. The results found are used to determine the Grüneisen parameters. This is to obtain the acoustic mode contribution to thermal expansion. After subtraction of the relaxation and anharmonic contributions, the temperature dependence of the shear wave ultrasound velocity follows a linear law as predicted by the Soft Potential Model.
\end{abstract}

Keyword: Acoustic; Metaphosphate glasses; Thermal expansion 